WIENER SLAVISTISCHES JAHRBUCH, Band 55/2009, 67-84

(C) 2009 by Österreichische Akademie der Wissenschaften, Wien

\author{
KARMEN PETRA MOISSI
}

\title{
In Wien gedruckte Bulgarica des 19. Jahrhunderts im Bestand der Österreichischen Nationalbibliothek (ÖNB)
}

\section{Die Wiener Slavica des 19. Jahrhunderts}

Die Wiener Druckereigeschichte verzeichnet für das 19. Jahrhundert erfreulicherweise auch einige Druckereien, die sich um die Verbreitung von Slavica im Habsburgerreich verdient gemacht haben (so z. B. die Unternehmen Karoline Pichler, Carl Ueberreuter, Leopold Sommer, Janko Kovačev, die Druckerei der Wiener Mechitharisten-Congregation oder die Kartographische Anstalt Freytag \& Berndt), wobei den Wiener Mechitharisten mit ihrer vielsprachigen Schulbücherproduktion vor allem für den Gymnasialbereich der Monarchie hier sicherlich eine Sonderstellung zukam. Die verlegerische Tätigkeit der Mechitharisten beruhte auf einem Ministerialerlass bzw. einem damit in Zusammenhang stehenden Vertrag mit der k. k. Schulbücherverschleißadministration ab 1. Jänner 1854 (Wytrzens 1985: 5-6; Mayer 1887: 185), dem ein Exklusivvertrag der Behörden mit den Wiener Druckereien Ueberreuter und Pichler aus dem Jahre 1844 über die Herstellung von Lehrbüchern für die Gymnasien vorangegangen war. Als 1853 schließlich den Unterrichtsbehörden die daraus entstehenden Kosten zu hoch erschienen, erhielt die Schulbücherverschleißadministration den Auftrag, nach kostengünstigeren Druckereien Ausschau zu halten. Als solche bot sich die Druckerei der Mechitharisten-Congregation an, die bis zu diesem Zeitpunkt ohnehin schon im Auftrag der beiden genannten Druckereien Ueberreuter und Pichler die Schulbuchproduktion übernommen hatte und somit bereits über kyrillische Lettern verfügte. Erfreulicherweise konnten sich die Mechitharisten in diesem Kontrakt auch das Recht erwirken, von jeder Neuauflage eines Schulbuches vier Belegexemplare zur Aufstellung in ihrer Bibliothek auf eigene Kosten drucken zu dürfen.

Es versteht sich von selbst, dass das in einer katholischen Ordensdruckerei gedruckte Schrifttum vor allem religiös orientiert war; die Mechitharisten-Druckerei zeichnete sich dennoch durch ungewöhnliche religiöse und nicht zuletzt politische Toleranz bei der Annahme von Druckaufträgen aus (es finden sich neben den katholischen Drucken auch orthodoxe und protestantische), und vor allem nach 1918 pro- 
duzierte sie rein kommerziell. So wurde bei den Mechitharisten unter anderem eines der frühesten bulgarischen Periodika, die Zeitschrift „Mirozrenie“ aus den Jahren 1850/51 (heute in Österreich nur noch im Bestand der Fachbibliothek des Instituts für Zeit- und Osteuropäische Geschichte ${ }^{1}$ ) gedruckt; hervorzuheben sind auch eine Darstellung der bulgarischen (Religons-)Geschichte aus dem Jahre 1861 (im Bestand der UB Wien ${ }^{2}$ ), ein Kalender für die Banater Bulgaren aus dem Jahre 1869 (im Bestand der ÖNB, Abb. 2) sowie ein Offizien-Buch von Ivan N. Momčilov ebenfalls aus dem Jahre 1869 (im Bestand der Bibliothek der Mechitharisten-Congregation und der ÖNB). Hier handelt es sich wahrlich um ein Kuriosum, findet sich darin doch eine Evangelien-Lesung in kirchenslawischer, neubulgarischer, griechischer, türkischer, rumänischer und französischer Sprache, die drei letztgenannten Sprachen gedruckt mit ornamental-kyrillischen Lettern (Wytrzens 1985: 14, 44) (Abb. 8).

\section{Die Wiener Bulgarica Des 19. Jahrhunderts}

In der Geschichte des Buchdrucks bildete das Osmanische Reich - und damit das bis 1878 in seinem Herrschaftsbereich gelegene Bulgarien - eine Ausnahme: Hier begann sich der Buchdruck erst Anfang des 18. Jahrhunderts zu etablieren ${ }^{3}$, aber noch bis ins 19. Jhdt. galt das strikte Verbot, islamische Religionstexte auf Arabisch zu drucken, wobei sich allerdings Druckerzeugnisse in anderen Schriften aus diesem geographischen Raum durchaus schon früher nachweisen lassen: In Konstantinopel und Saloniki, den zunächst beiden einzigen Druckorten des Reiches, druckten bereits ab 1493 aus Spanien und Portugal vertriebene Juden hebräische Texte, und es entstand so neben Amsterdam und Venedig ein drittes Zentrum jüdischer Buchkultur. Auch im Libanon war der Druck christlicher Texte ab dem 17. Jhdt. in geringem Umfang möglich. Erst im 19. Jahrhundert nahm die Zahl der Druckerzeugnisse und damit auch der Umfang der Bibliotheksbestände im Osmanischen Reich merklich $\mathrm{zu}$, wenngleich diese auch weiterhin nur einer Bildungselite zugänglich blieben (Stein 2006: 195).

Wollten also bulgarische Autoren Anfang des 19. Jahrhunderts ihre Werke drucken lassen, mussten sie sich vorzugsweise an Druckereien außerhalb Bulgariens wenden, da Bulgarien aus den bereits erwähnten Gründen kaum über moderne Druckereien verfügte (eine der wenigen - übrigens mit Wiener Druckpressen ausgerüsteten - leistungsfähigen Offizinen befand sich im Rila-Kloster [Wytrzens 1982: 125]). Als Druckorte außerhalb Bulgariens sind in diesem Zusammenhang neben

${ }^{1}$ Mirozrenie. Spisánie pomésjačno. Sočinitel Ioánn Dóbrovič. I (1850/51) 1-5. 84 (durchgez.) S. Sign.: 20.119 a O, adl. 5. Mehr wurde in Wien nicht publiziert; eine 2. Serie erschien 1870 in Bukarest. Wytrzens 1985: 46-48.

2 Osmotrenija na istorijata bălgarskago naroda. 1861. 16 S. Sign.: I 403.917. Wytrzens 1985: 49.

3 Der Buchdruck wurde erst 1727 unter Ahmet III. eingeführt. Wikipedia: Ahmet_III und Türk ans. 1952: 334; Kemke 1894; Weil 1907; Chauvin 1907. 
Konstantinopel die ost- bzw. südosteuropäischen Städte Bukarest, Rimnik, Budapest, Belgrad, Saloniki, Moskau und Odessa, aber auch London, Rom und nicht zuletzt Wien zu nennen.

Es gibt vorrangig wohl zwei Gründe, weshalb Wien als Druckort für die Bulgaren attraktiv gewesen sein könnte: Zum einen agierten die Wiener Druckereien sehr kommerziell und weltanschaulich tolerant bei der Annahme von Druckaufträgen (wobei sich die Situation für die Bulgaren noch verbesserte, als Kovačev und Danov in den Jahren 1874-1878 - zunächst am Schmerlingplatz, ab 1875 in der Florianigasse 48 - eine eigene Druckerei betrieben und die Mehrzahl der bulgarischen Aufträge an sich ziehen konnten), zum anderen liegt die Vermutung nahe, dass die Wiener Zentralstellen angesichts der politischen Entwicklung auf dem Balkan die Publikationstätigkeit bulgarischer Kulturpioniere in Wien wachsam und durchaus wohlwollend verfolgten, um so (kultur-)politisch geschickt die Weichen für die Zeit nach dem sich bereits abzeichnenden Zusammenbruch des Osmanischen Reiches stellen zu können.

Die Wiener Bulgarica boten Informationen über die Bulgaren und ihr Land, dessen Geschichte sowie über dortige aktuelle Ereignisse: Es finden sich erstaunlich viele Publikationen, die die Volkswirtschaft Bulgariens zum Thema haben, daneben erschien aber auch Erbauungsliteratur, in der zu Fragen der Ethik und der Lebensführung Stellung genommen wurde. Sehr umfangreich war die Zahl der Bücher über religiöse Themen, deren Großteil aus nahe liegenden Gründen in der Mechitharisten-Druckerei hergestellt wurde. Die Mehrzahl der Wiener Bulgarica bildeten jedoch Sach- bzw. Lehrbücher für verschiedene Altersgruppen, die die Bereiche Wissenschaft und Volksbildung abdeckten. Man denke nur an Christo Danov, Dragan Mančev, Ivan Momčilov, Joakim Gruev, deren Publikationen aus der Druckerei Leopold Sommer \& Co. ${ }^{4}$ vorwiegend an der Österreichischen Nationalbibliothek zu finden sind (Wytrzens 1982: 130-131). Darüber hinaus wurden in Wien zahlreiche Bücher, Broschüren, Periodika und Landkarten (letztere erschienen Ende des 19. Jhdts. übrigens alle ausnahmslos bei Freytag \& Berndt) für die Bulgaren selbst gedruckt. Eine nicht zu unterschätzende Bedeutung kam während der „Wiedergeburtszeit“ auch der heute kaum noch bekannten bulgarischen Organisation „Napredăk“ zu, die in Wien wirkte und sich sowohl politische als auch volksbildnerische Ziele gesetzt hatte. Diese Organisation publizierte Statuten und Jahresberichte, die heute in Wien leider nicht mehr zu finden sind (Wytrzens 1982: 125).

Während der „Wiedergeburtszeit““ war die Verlagstätigkeit der Druckereien Kovačev und Sommer im Gegensatz zu jener der Mechitharisten rein marktorientiert, und diese kapitalistische Einstellung wirkte sich - wie Wytrzens in seinem Aufsatz über die Bedeutung Wiens für die bulgarische ,Wiedergeburtszeit““ ironisch bemerk-

${ }^{4}$ Zur Geschichte dieser Druckerei siehe Mayer 1887: 229-232. Vgl. dazu auch Popov 1994: 163 ff. 
te - „keineswegs zum Schaden für die bulgarische Kultur“ aus (Wytrzens 1982: 132). Recht anschaulich offenbart sich an den südslawischen Drucken die allmähliche Herausbildung einer allgemein anerkannten Rechtschreibung bzw. einer stabilen Sprach- und Schriftform. Gerade die Mechitharisten-Druckerei bot hier ausreichend Gelegenheit zum Experimentieren, da sie keiner der orthographischen oder grammatischen „Schulen“ verpflichtet war und über einen reichen Vorrat an Schrifttypen verfügte, die teilweise sogar im Hause geschnitten wurden (Wytrzens 1984: 180).

Auch am Aufbau des bulgarischen Schulwesens hatten die Wiener Druckereien durchaus ihren Anteil. Als Auftraggeber fungierten bekannte bulgarische Kulturpioniere und für die bulgarische Befreiungsbewegung bedeutsame Persönlichkeiten ${ }^{5}$ wie etwa der bereits erwähnte Christo G. Danov, der vorrangig bei Leopold Sommer publizierte und - wie bereits erwähnt - gemeinsam mit Janko Kovačev in Wien von 1874 bis 1878 eine Druckerei betrieb, oder Ivan N. Momčilov, Ivan Dobrovski (Ioan Dobrovič), Dragan V. Mančev (Mančov) und Najden P. Stojanov, die sowohl an die Druckerei Sommer als auch an die Mechitharisten Druckaufträge vergaben. Sommer hatte mit 274 Titeln den größten Anteil an der Produktion bulgarischer Werke (Wytrzens 1982: 129).

III. Die Wiener Bulgarica des 19. Jahrhunderts im Bestand der ÖSterReichischen NATIONALBiBLIOTHEK

Erstaunlich akribisch erfasst ist das in Wien erschienene bulgarische Schrifttum in der Bibliographie von Man'o Stojanov betreffend die Literatur der „Wiedergeburtszeit" - sie verzeichnet für den Zeitraum 1806-1878 rund 8900 Titel (Stojanov 1957). Als wertvoll erweisen sich darüber hinaus die Bibliographien von Dimităr Ivančev, Valerij Pogorelov und Aleksandăr Balan ${ }^{6}$. Und nicht zuletzt ist in diesem Zusammenhang auch (Josef) Konstantin Jirečeks Bibliographie der neubulgarischen Buchproduktion von 1806-1870 zu nennen, die 1872 bei Leopold Sommer erschien ${ }^{7}$ (alle diese Bibliographien finden sich im Bestand der ÖNB). Heute zählen Konstantin Jireček und Felix Kanitz zu jenen österreichischen Gelehrten, die sich um die Verbreitung von Wissen über Bulgarien in der Habsburgermonarchie besondere Verdienste erworben haben.

Als zusätzliche Arbeitsgrundlage für den vorliegenden Beitrag dienten die Nachschlagewerke von Georgi Boršukov (Boršukov 1976) und Dimităr Ivančev (Ivančev

${ }^{5}$ Zum Thema „Bulgarische Kultur während der ,Wiedergeburtszeit'“ erschien im Jahre 1988 am Institut für Geschichte der Bulgarischen Akademie der Wissenschaften eine umfangreiche Bibliographie mit 6190 Einträgen, die einen Berichtszeitraum von 105 Jahren umfasst: Gečeva 1986.

6 Ivančev 1978-1995 (55.851 Einträge); Pogorelov 1923 (1623 Einträge); Balan 1909 (15.258 Einträge).

7 Jireček 1872 (532 Einträge +18 im Anschluss ergänzte Titel). 
1962-1969) sowie fallweise auch Informationen aus der „Enciklopedija Bălgarija“ (Enc. Bălg. 1978-1996).

Einen guten Überblick über die Bulgarica des 19. Jahrhunderts an der Österreichischen Nationalbibliothek bietet ein erstes von sechs Bulgaristik-Studentinnen unter der Leitung von Ljubka Lipčeva-Prandževa im WS 2005/06 am Institut für Slawistik der Universität Wien erstelltes Verzeichnis dieses speziellen Bestandes an unserer Bibliothek (Radeva 2006: 18). Natürlich kann - wie bei den meisten Bibliographien - auch hier nicht der Anspruch auf Vollständigkeit erhoben werden. Die teilweise fehlerhaften Katalogisate der in der erwähnten Bibliographie der Studentinnen verzeichneten Drucke im Katalog 1501-1929 der ÖNB wurden inzwischen nach Autopsie korrigiert und sind nun für den Bibliotheksbenützer problemlos suchbar.

Das chronologische und nach Druckereien gegliederte Verzeichnis der Wiener Bulgarica des 19. Jahrhunderts befindet sich bereits im Bestand der Österreichischen Nationalbibliothek und steht somit den Leserinnen und Lesern des Hauses zur Verfügung. Es umfasst insgesamt 77 Druckschriften aus dem Bestand Neu (Standort: Heldenplatz) sowie 33 geographische Karten aus dem Bestand der Kartensammlung der NB. Diese Karten aus den Jahren 1890-1900 waren entweder Teillieferungen größerer Atlaswerke (siehe Abb. 1) oder dienten als Lehrbehelfe für den bulgarischen Geographieunterricht - unter ihnen finden sich nicht wenige Wandkarten.

Die an der Österreichischen Nationalbibliothek vorhandenen bulgarischen bzw. kirchenslawisch-bulgarischen Druckschriften des 19. Jahrhunderts entstanden zum kleineren Teil in der Mechitharisten-Druckerei (6 Titel) (Moissi 1999: 47), bei Kovačev (13) und Holzhausen (1), zum größten Teil jedoch - wie bereits erwähnt - in der Druckerei Sommer (56). Stojanov verzeichnet in seiner Bibliographie Auflagenhöhen von etwa 300-1000 Exemplaren pro Auflage.

Höchst erfreulich für die ÖNB ist die hohe Zahl an abgelieferten Druckschriften aus der Druckerei Sommer sowie an Karten aus der Kartographischen Anstalt Freytag \& Berndt, war doch im 19. Jahrhundert die Moral bezüglich der Pflichtablieferungen noch nicht sehr ausgeprägt. Zwar gab es bereits das so genannte „Pflichtexemplar" (Strebl 1970), dessen Nachweis noch heute eine der Hauptaufgaben der NB darstellt, dennoch schaffte man es trotz strengster Vorschriften der Bücherrevisionsämter nicht, alle Drucke der Habsburgermonarchie lückenlos zu erfassen. So waren es wohl vorrangig politische Gründe, Informationen über die nationalen Entwicklungen in den Kronländern nur sehr gefiltert in die Reichshauptstadt dringen zu lassen; andererseits verstand die Hofbibliothek des 19. Jahrhunderts ihren Sammelauftrag anders als die heutige Nationalbibliothek: Während im 19. Jahrhundert durch die Hofbibliothekare eine strenge Selektion der abgelieferten Literatur erfolgte und ausgeschiedene Literatur auf dem Tauschwege oder gar über den Altpapierhandel entsorgt wurde, ist die Nationalbibliothek heute bemüht, die Pflichtexemplare möglichst lückenlos und wertfrei zu sammeln bzw. nachzuweisen, und sieht sich so nicht selten in der Situation, Austriaca - einst ,ausgeschieden“, weil damals „unter allen 
Umständen und in jeder Hinsicht wertlos" - antiquarisch um erkleckliche Summen wieder ankaufen zu müssen (Hüttl-Hubert 2004: 96-98).

$\mathrm{K}$ u r z p o r trä t s einiger dieser kulturpolitisch - und daher meist auch politisch - sehr engagierten Autoren bzw. Herausgeber, deren Werke in den Bestand der ÖNB aufgenommen wurden, mögen zum besseren Verständnis der damaligen Umund Aufbruchstimmung im Bulgarien der „Wiedergeburtszeit“ und vor allem später während der Befreiungskämpfe beitragen ${ }^{8}$ :

Eine der schillerndsten Persönlichkeiten im öffentlichen Leben Bulgariens war sicherlich Christo G. Danov (geb. 1828 in Klisura, gest. 1911 in Plovdiv). Neben seiner Tätigkeit als Lehrer und Buchhändler gilt er bis heute als der Initiator organisierter verlegerischer Tätigkeit in Bulgarien. Bereits 1857 gründete er in Plovdiv eine Verlagsbuchhandlung unter dem Namen „Christo Danov \& Co.“, als seine Teilhaber fungierten Jako Truvčev und Joakim Gruev. 1867 entstanden Zweigstellen in Ruse und Veles, 1880 gab es bereits Filialen in Sofija und Lom. 1874 gründeten Danov und Kovačev eine bulgarische Druckerei in Wien, in der innerhalb von zwei Jahren etwa 50 bulgarische Bücher gedruckt wurden (Bălg. enc. 1999: 507) (als Beispiel siehe Abb. 3). 1878 wurde diese auf Wunsch von Fürst Aleksej M. DondukovKorsakov nach Plovdiv verlegt, und 1879 eröffneten sie mit der materiellen Unterstützung von Dondukov-Korsakov eine eigene Druckerei in Sofija. 1881 schließlich trennte sich Danov von Kovačev und weitete seine verlegerische Tätigkeit noch aus. Alles in allem erschienen bei Danov insgesamt etwa 900 Publikationen (Lehrbücher, Lehrbehelfe, wissenschaftliche Werke und Kunstdrucke). Auch politisch zeigte er sich engagiert: 1882 fungierte er als Abgeordneter zur III. Einfachen Volksversammlung, 1896-1899 hatte er das Amt des Bürgermeisters in Plovdiv inne. Im Jahre 1900 wurde er schließlich zum Ehrenmitglied der Bulgarischen Akademie der Wissenschaften ernannt ${ }^{9}$.

Einer der Teilhaber Danovs war Joakim P. Gruev (1828-1912). Er kodifizierte bereits 1858 als Vertreter der „Plovdiver Schule“ mit seinem von Christo Danov und Jako Truvčev herausgegebenen Werk „Osnova za bălgarska gramatika“ (Abb. 4) ein Muster für eine zukünftige Literatursprache (die 2., überarb., und die 3. Auflage erschienen 1862 bzw. 1864, die 4. Aufl. 1865, die 5. Aufl, - fälschlich ebenfalls als 4. Aufl. bezeichnet - 1869 bei Sommer in Wien [alle im Bestand der ÖNB]).

${ }^{8}$ Bei diesen Kurzporträts stütze ich mich - soweit überhaupt Informationen gefunden werden konnten und keine anderen Quellen zitiert werden - auf die Angaben in den entsprechenden Nationalenzyklopädien (Enc. Bălg. 1978-1996, Enc. Jug. 1955-1971; Eleutherudakes 1927-1931) sowie auf kurze biographische Daten bei Stojanov 1957. In den Enzyklopädien findet sich meist auch weiterführende autobiographische, biographische bzw. Sekundärliteratur zu den einzelnen Persönlichkeiten zitiert. Darüber hinaus vgl. auch Vasiliev 1995.

9 Dimitrov 1988. Der Band erschien im Verlag „Christo G. Danov“; auf der Titelseite ist als Gründungsjahr des Verlags das Jahr 1855 angegeben. 
Auch Dobri P. Vojnikov (1833-1878) publizierte sein „Răkovodstvo za slovesnost", mit dem er die bulgarische Gegenwartssprache deutlich beeinflusste, unter der Herausgeberschaft von Christo Danov 1874 bei Sommer in Wien (im Bestand der ÖNB, Abb. 5). Er war Schriftsteller, Lehrer und Journalist und gilt heute als Begründer des bulgarischen Theaters, als Mitbegründer der Bulgarischen Literarischen Gesellschaft (Bălgarsko knižovno družestvo, BKD) sowie als erster bulgarischer Regisseur. Politisch verfolgt, emigrierte er nach Rumänien und gründete in Brăila die erste bulgarische Theatertruppe. Darüber hinaus unterrichtete er Musik und Deklamation sowie Notenlehre, und auch als Herausgeber von Schulbüchern machte er sich einen Namen.

In diesem Zusammenhang ist auch Ivan A. Bogorov (Bogoev) (ca. 1820-1892) zu nennen. Wie Vojnikov war er Literat, Journalist und Sprachforscher, ebenfalls Gründungsmitglied der Bulgarischen Literarischen Gesellschaft, wie Vojnikov bemühte auch er sich um die Schaffung einer neubulgarischen Standardsprache (Abb. 7). 1842 publizierte er den ersten Band bulgarischer Volkslieder, wurde jedoch vor allem durch seine Übersetzungen und die Herausgabe von Wörterbüchern bekannt.

Ähnlich engagiert, allerdings vor allem im kirchenpolitischen Bereich, zeigte sich der Lehrer und Schriftsteller Ivan N. Momčilov (geb. 1819 in Elena, gest. 1869 in Gorna Orjachovica), der einige seiner Werke bei Sommer bzw. bei den Mechitharisten drucken ließ (Abb. 8). Seine Studien absolvierte er in Griechenland (u. a. bei Theophilos Kairis) und Russland und kämpfte nach seiner Rückkehr für die Unabhängigkeit der bulgarischen Kirche unter osmanischer Herrschaft. Nach seiner Tätigkeit als Lehrer in Elena und Gorna Orjachovica begann er als Schriftsteller zu arbeiten und trat vehement für die Einführung des Neubulgarischen anstelle des Kirchenslavischen im Unterricht ein, wodurch ihm auch bei der Entwicklung des neubulgarischen Bildungswesens große Verdienste zukommen. Darüber hinaus wurde auf seine Initiative hin erstmals „Gymnastik“ als Unterrichtsfach eingeführt. Viele seiner Schüler wählten später ebenfalls den Beruf des Lehrers und waren aktiv an den Befreiungskämpfen beteiligt (Petko Slavejkov, Dragan Cankov, Nikola Kozlev, Ivan Kasabov u. a.). In vielerlei Hinsicht trug Momčilov zur Verbreitung von Literatur verschiedenster Art bei, verfasste, verlegte und übersetzte Lehrbücher und war Autor von Grammatiken der altbulgarischen bzw. der neubulgarischen Sprache ${ }^{10}$, deren erste bzw. zweite Auflage bei Sommer in Wien erschien.

Auch Dragan (Dimităr) Vasilev Mančev (Mančov) (geb. 1824 in Batak, gest. 1908 in Plovdiv) war Schriftsteller, Verleger und Politiker. Bereits im Alter von sechzehn Jahren begann er zu unterrichten, 1854 erschien in Konstantinopel sein erstes Buch mit dem Titel „Cărkovno pesnopenie, săbrano i săčineno ot razni pevci“ mit kirchenslavischem Text und griechisch-byzantinischen Noten. 1862 gab er seinen Lehrerberuf schließlich auf und eröffnete eine Buchhandlung in Plovdiv mit

${ }^{10}$ Momčilov 1865; Momčilov 1868 (2. Aufl. Wien 1873). 
Filialen in Svištov und Saloniki. Als Herausgeber von Schulbüchern ließ er seine Bücher zunächst in Konstantinopel, dann in Brăila und Wien drucken (Abb. 6). Schließlich plante er die Einrichtung einer eigenen Druckerei in Plovdiv, wurde jedoch von der osmanischen Verwaltung der Beteiligung am Aufstand von Starozagorsk 1875 beschuldigt und arretiert. Nach zwei Monaten freigelassen, flüchtete er 1876 nach Bukarest, übernahm dort Christo Botevs Druckerei und publizierte in den Jahren 1876-77 unter dem Pseudonym „D. Veselinov“ die von Stefan Bobčev redigierte Zeitung „Stara planina“. Sobald die Russen die Donau überschritten hatten, verkaufte er seine Drucklettern und kehrte nach Plovdiv zurück. Von der St. Petersburger „Slawischen Wohltätigkeitsgesellschaft“ (Slavjanskoe blagotvoritel'noe obščestvo) ${ }^{11}$ erhielt er eine russische Schnellpresse mit etwa $800 \mathrm{~kg}$ an Drucklettern und begann mit der Herausgabe der Zeitung „Narodnij glas“. Ständig erweiterte er seine Ausstattung und entwickelte eine sehr rege verlegerische Tätigkeit - bis 1908 erschienen in Mančevs Druckerei etwa 450 Bücher, unter ihnen auch die erste bulgarische Enzyklopädie, der „Enciklopedičeski rečnik“ von Luka Kasărov. Die Druckerei wurde nach seinem Tode von Mančevs Erben verkauft.

\section{ZUSAMMENFASSUNG}

Vor allem die vor 1878 erschienenen Wiener Bulgarica vermitteln in ihrer Vielfalt ein facettenreiches Bild der volksbildnerischen, kultur- und religionspolitischen Bestrebungen der Bulgaren, sich schrittweise vom Osmanischen Reich zu emanzipieren und eine eigene nationale Identität zu schaffen. Nicht wenige Beispiele dafür finden sich erfreulicherweise im Bestand der Österreichischen Nationalbibliothek und warten noch auf ihre Erforschung im gesamteuropäischen Kontext. In der von der Bulgarischen Nationalbibliothek präsentierten Ausstellung „In Wien gedruckte bulgarische Bücher 1845-1878“, die in Kooperation mit der ÖNB im Prunksaal der Österreichischen Nationalbibliothek im Februar 2007 gezeigt wurde, konnte dieser besondere Bestand der ÖNB erstmals einer breiteren Öffentlichkeit vorgestellt wer$\operatorname{den}^{12}$.

\section{L it e r a t u r}

Bachmaier 1998: $\quad$ P. Bachmaier, Der Panslawismus in Bulgarien von 1878 zur Gegen-

Balan 1909: $\quad$ A. T. Balan (ed.): Bălgarski knigopis za sto godini. 1806-1905. Matewart, Die slawischen Sprachen 56, 25-39

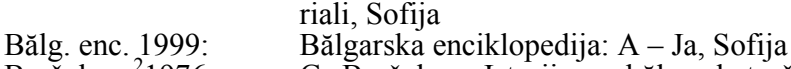

Boršukov ${ }^{2} 1976$ : $\quad$ G. Boršukov, Istorija na bălgarskata žurnalistika. 1844-1877. 18781885, Sofija

11 Zur Bedeutung der „Slawischen Wohltätigkeitsgesellschaft“ vgl. Bachmaier 1998: 25-39.

12 Kirilova 2007. 
Chauvin 1907: $\quad$ V. Chauvin, Notes pour l'histoire de l'imprimerie à Constantinople, Zentralblatt für Bibliothekswesen 24, 255-262

Dimitrov 1988: $\quad$ A. Dimitrov, Knižarjat, kogoto naričacha ministăr, Plovdiv

Eleutherudakes 1927-1931: K. Eleutherudakes, Enkyklopaidikon lexikon, Athen

Enc. Bălg. 1978-1996: Enciklopedija Bălgarija, Sofija

Enc. Jug. 1955-1971: Enciklopedija Jugoslavije, Zagreb

Gečeva 1986:

Hüttl-Hubert 2004:

K. Gečeva, Bălgarskata kultura prez Văzraždaneto. Bibliografija. Bălgarska i čužda knižnina 1878-1983, Sofija

Ivančev 1962-1969: D. Ivančev (ed.): Bălgarski periodičen pečat 1844-1944. Anotiran E. Hüttl-Hubert, Mehr als ein Ort der Erinnerung: Die Österreichische Nationalbibliothek und ihre Slavica, Biblos 53/2, 93-108

Ivančev 1978-1995: D. Ivančev (ed.), Bălgarski knigi 1878-1944. Bibliografski ukazatel. Azbučna poredica, Sofija

Jireček 1872:

Kemke 1894: J. Konst(antin) Jireček (ed.): Knigopis na novobălgarska-ta knižnina 1806-1870, Viena

Kirilova 2007:

J. Kemke, Zur Geschichte des Buchdrucks in Constantinopel, Centralblatt für Bibliothekswesen 11, 178-184

R. Kirilova (ed.), Bălgarski knigi pečatani văv Viena 1845-1878 = In Wien gedruckte bulgarische Bücher 1845-1878, Sofija

Mayer 1887:

Moissi 1999:

Momčilov 1865:

Momčilov 1868:

Pogorelov 1923:

A. Mayer, Wiens Buchdrucker-Geschichte 1482-1882, Bd. II: 16821882, Wien

K. P. Moissi, Die Bulgarica der Wiener Mechitharisten-Druckerei, Wien, ÖNB, Bibliothekar. Hausarb.

I. Momčilov, Gramatika za starobălgarskija jezik po sičkoto mu razvitie, Viena

I. Momčilov: Gramatika za novobălgarskija ezik, Rusčuk

V. Pogorelov (ed.), Opis na starite pečatani bălgarski knigi (18021877 g.), Sofija

Popov 1994:

Radeva [2006]:

Stein 2006:

Strebl 1970:

D. Popov, Dobri Vojnikov und seine Redekunsttheorie aus der Zeit der bulgarischen Wiedergeburt, Wiener Slavistisches Jahrbuch 40, $163-168$

A. Radeva et al., Bălgarski knigi, pečatani văv Viena prez XIX-ti vek, săchranjavani v Avstrijskata nacionalna biblioteka, Manuskript, Viena P. Stein, Schriftkultur. Eine Geschichte des Schreibens und Lesens, Darmstadt

M. Strebl, Die Wiener Hofbibliothek und die Pflichtexemplare, in: Josef Mayerhöfer et al. (ed.), Festschrift Josef Stummvoll, dem Generaldirektor der Österreichischen Nationalbibliothek zum 65. Geburtstag / 19. August 1967 dargebracht von seinen Freunden und Mitarbeitern, Wien (= Museion, N.F., 2,4), 348-356

Stojanov 1957: $\quad$ M. Stojanov (ed.), Bălgarska văzroždenska knižnina. Analitičen repertoar na bălgarskite knigi i periodični izdanija. 1806-1878, T. I, Sofija

Türk ans. 1952:

Vasiliev ${ }^{2}$ 1995:

Weil 1907:

Wikipedia:

Wytrzens 1982:

Wytrzens 1984:

Türk ansiklopedisi, Cilt V, Ankara

A. Vasiliev, Trěm na bălgarskoto Văzraždane, Sofija

G. Weil, Die ersten Drucke der Türken, Zentralblatt für Bibliothekswesen 24, 49-61

http://de.wikipedia.org/wiki/Ahmet_III (Zugriffsdatum: 23.6.2008)

G. Wytrzens, Die Bedeutung Wiens und seiner Druckereien für die bulgarische Wiedergeburt, Die slawischen Sprachen 1, 125-133

G. Wytrzens, Die slavischen und slavenkundlichen Drucke der Wiener Mechitharisten, in: 13. ABDOSD-Tagung. Wien, 28.-30. Mai 1984. Referate und Beiträge. Berlin (= Staatsbibliothek Preussischer Kulturbesitz. Veröffentlichungen der Osteuropa-Abteilung, 3), 178-186

Wytrzens 1985: $\quad$ G. Wytrzens, Die Slavica der Wiener Mechitharisten-Druckerei, Wien 
A b stract: Bulgarian works printed in Vienna in the $19^{\text {th }}$ century now in the Austrian National Library $(\ddot{O} N B)$. During the Bulgarian Renaissance and up to the end of the $19^{\text {th }}$ century, Viennese publishers (e.g. Karoline Pichler, Carl Ueberreuter, Leopold Sommer, Janko Kovačev, the Mekhitharist Congregation of Vienna, the cartographic publisher Freytag \& Berndt) printed an impressive number of maps and Bulgarian books on education, cultural policy and religious affairs. Because of the strict rules for legal deposits within the Habsburg Monarchy, most of these printings can be found in the stocks of the Austrian National Library. These Bulgarian books give colourful evidence of the Bulgarian intellectual struggle against the Ottoman Empire, as most of the authors and editors belong to the famous Bulgarian intelligentsia who fought for freedom: Khristo G. Danov, Joakim P. Gruev, Dobri P. Voynikov, Ivan A. Bogorov, Ivan N. Momchilov, Dragan V. Manchev and Nayden P. Stoyanov.

In February 2007, the exhibition "Bulgarian books printed in Vienna 1845-1878" in the State Hall of the Austrian National Library afforded an opportunity to get acquainted with these unique deposits in the Austrian National Library.

K e y w ords: Vienna, printing and publishing, Bulgarian renaissance, Bulgarian printers, Bulgarian intellectuals, Bulgarian books, Bulgarian textbooks, Bulgarian periodicals, maps 

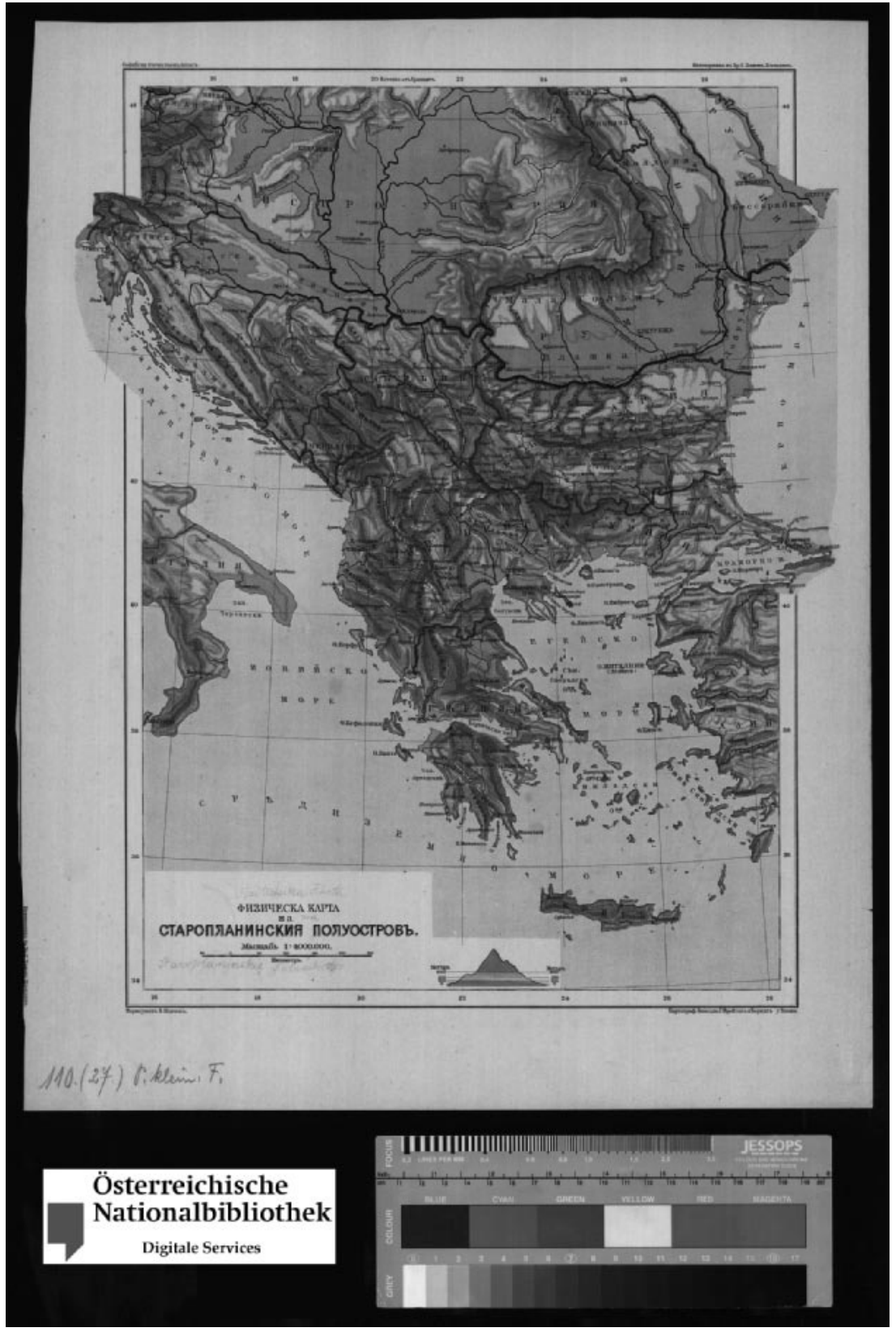

Abb. 1: Fizičeska karta na Staroplaninskija poluostrov. - Wien, Freytag \& Berndt 1899. Aus: Sofijski Otečestven Atlas. Sign.: AB 110 (27) Kar 


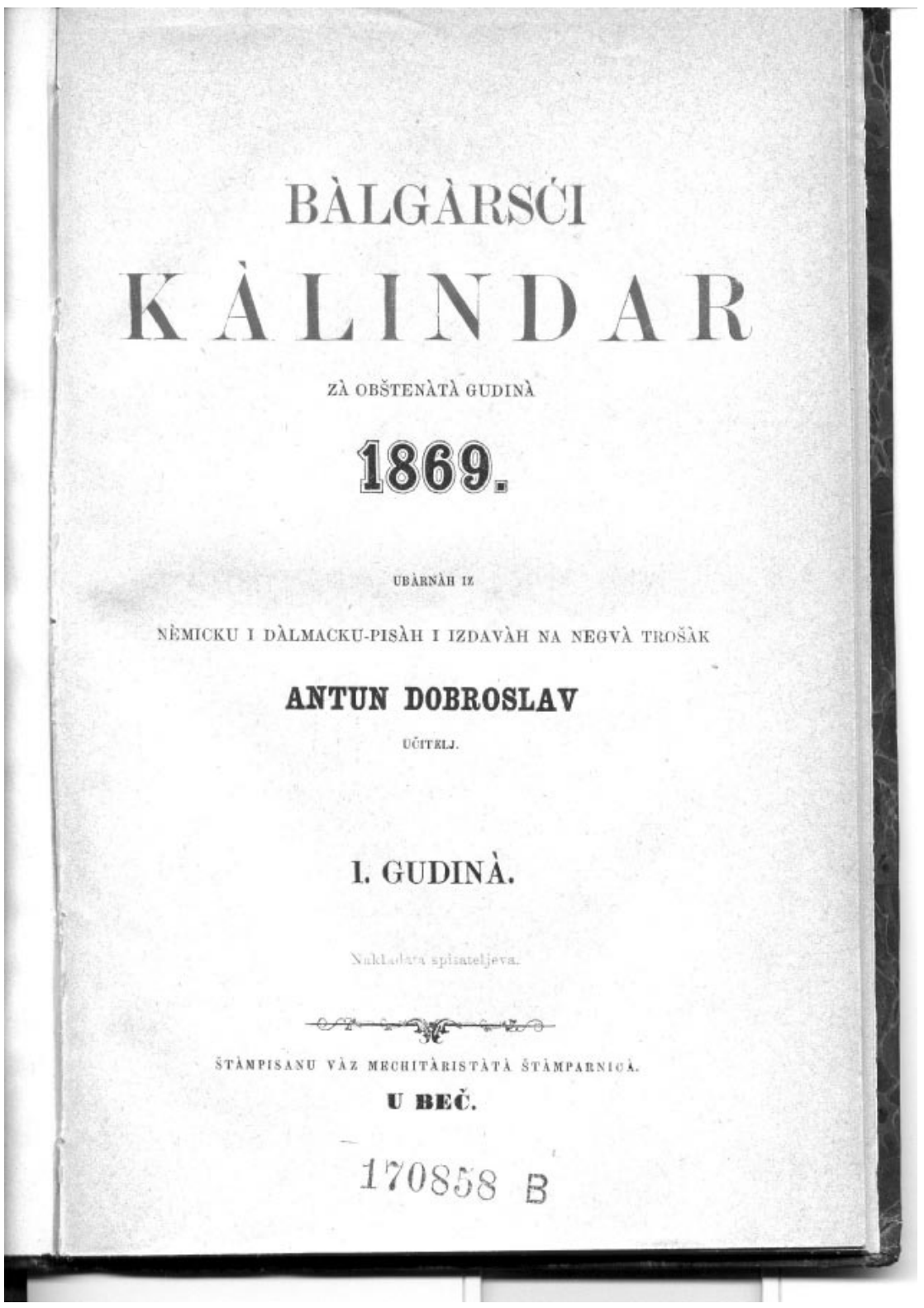

Abb. 2: Dobroslav, Antun: Bàlgàrsći Kàlindar zà obštenàtà gudinà 1869. - Wien, Mechitharisten 1869. 53 S. Sign: 170.858-B.Neu- 


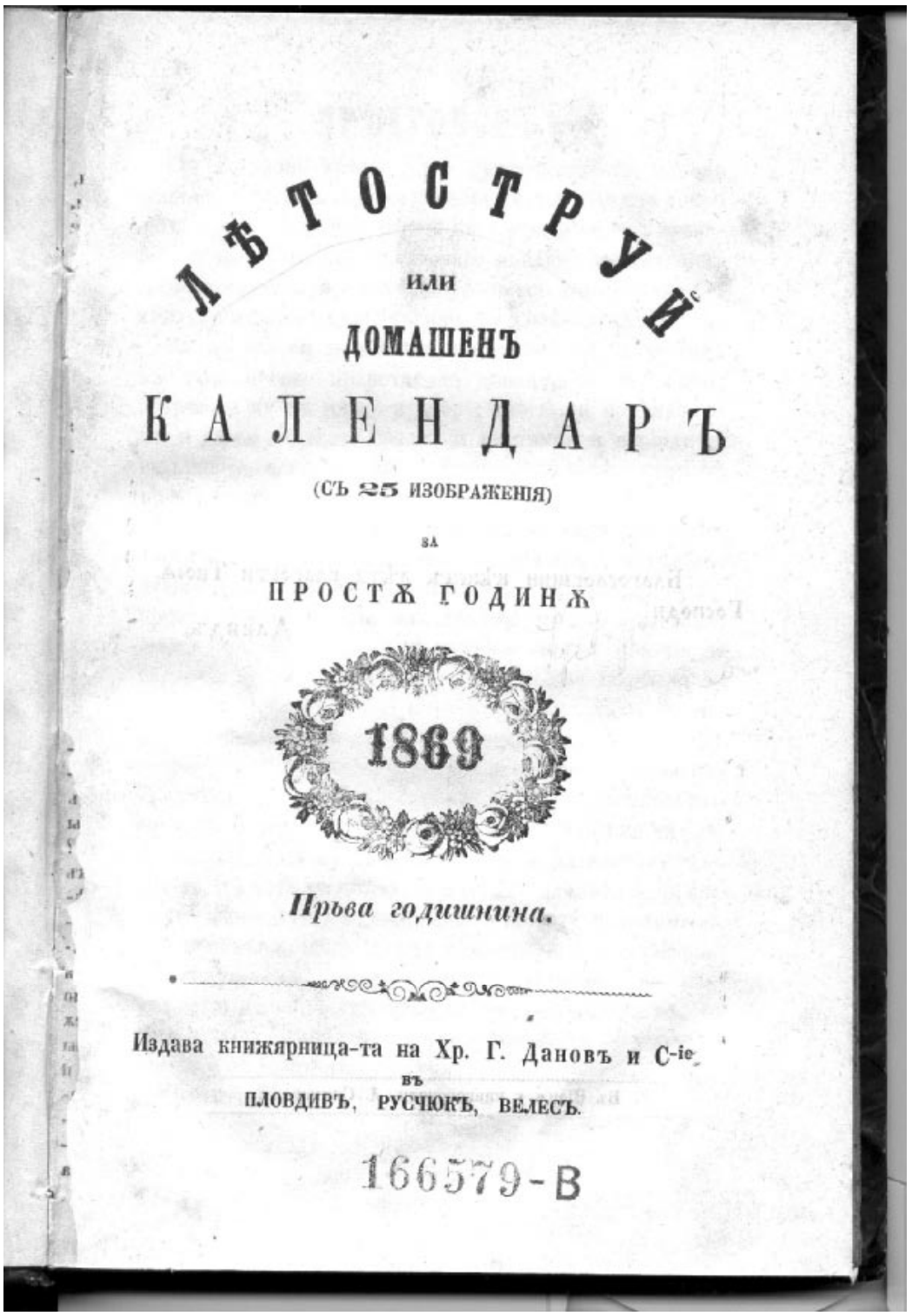

Abb. 3: Lětostruj ili domašen kalendar. - Plovdiv, Danov 1859-1876 ${ }^{13}$. Sign.: 166.579B.Neu-

${ }^{13}$ Ab Jg. 7.1875 unter dem Titel: Bălgarskij naroden kalendar. Bis Jg. 6.1874 Druckort und Drucker: Wien, L. Sommer, ab Jg. 7.1875 übernahm Kovačev die Produktion. 


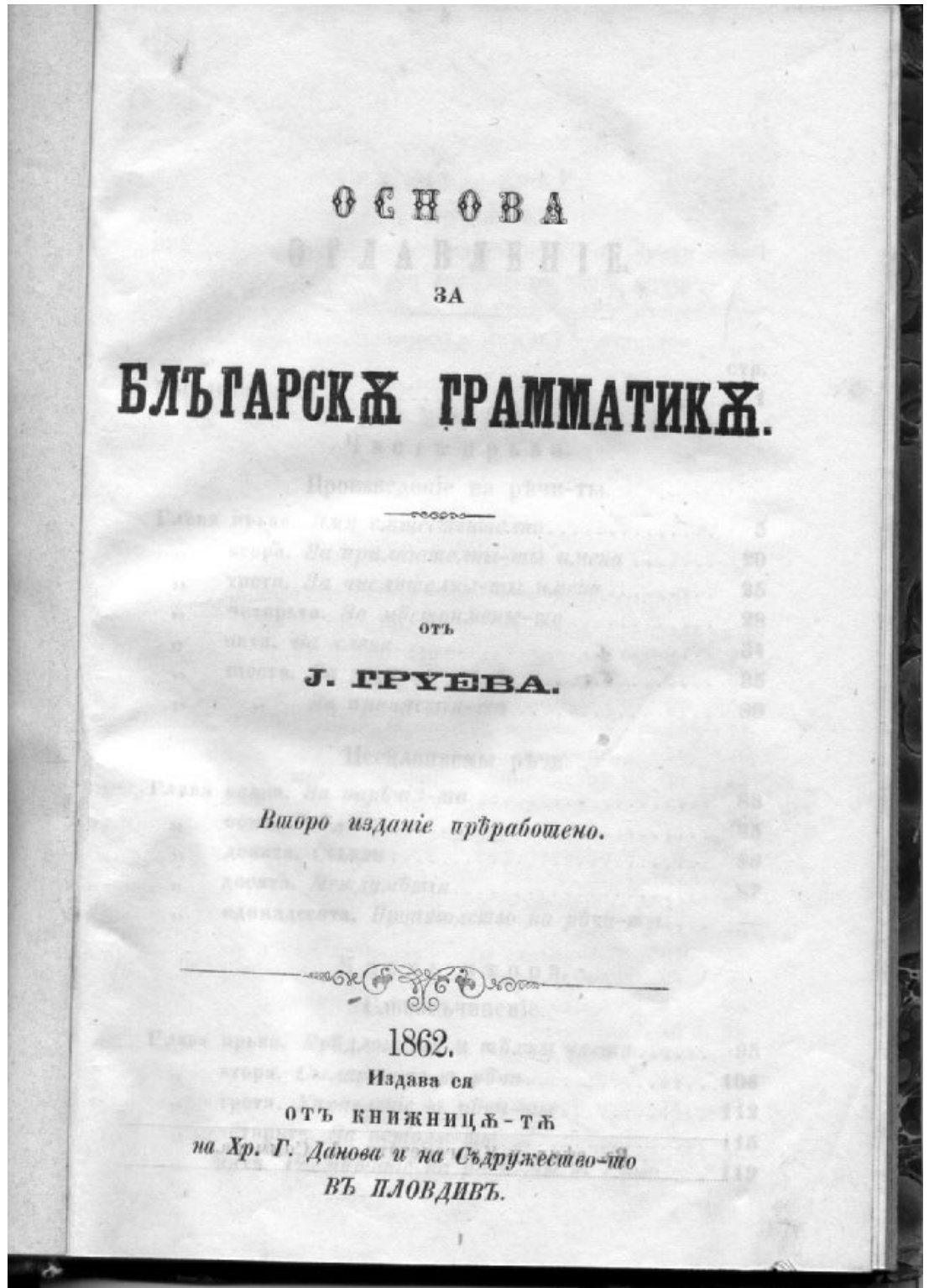

Abb. 4: Gruev, Joakim P.: Osnova za blăgarska grammatika ot J. Grueva. Plovdiv, Danov 1862. 144 S. Sign.: 98.650-B.Neu- 


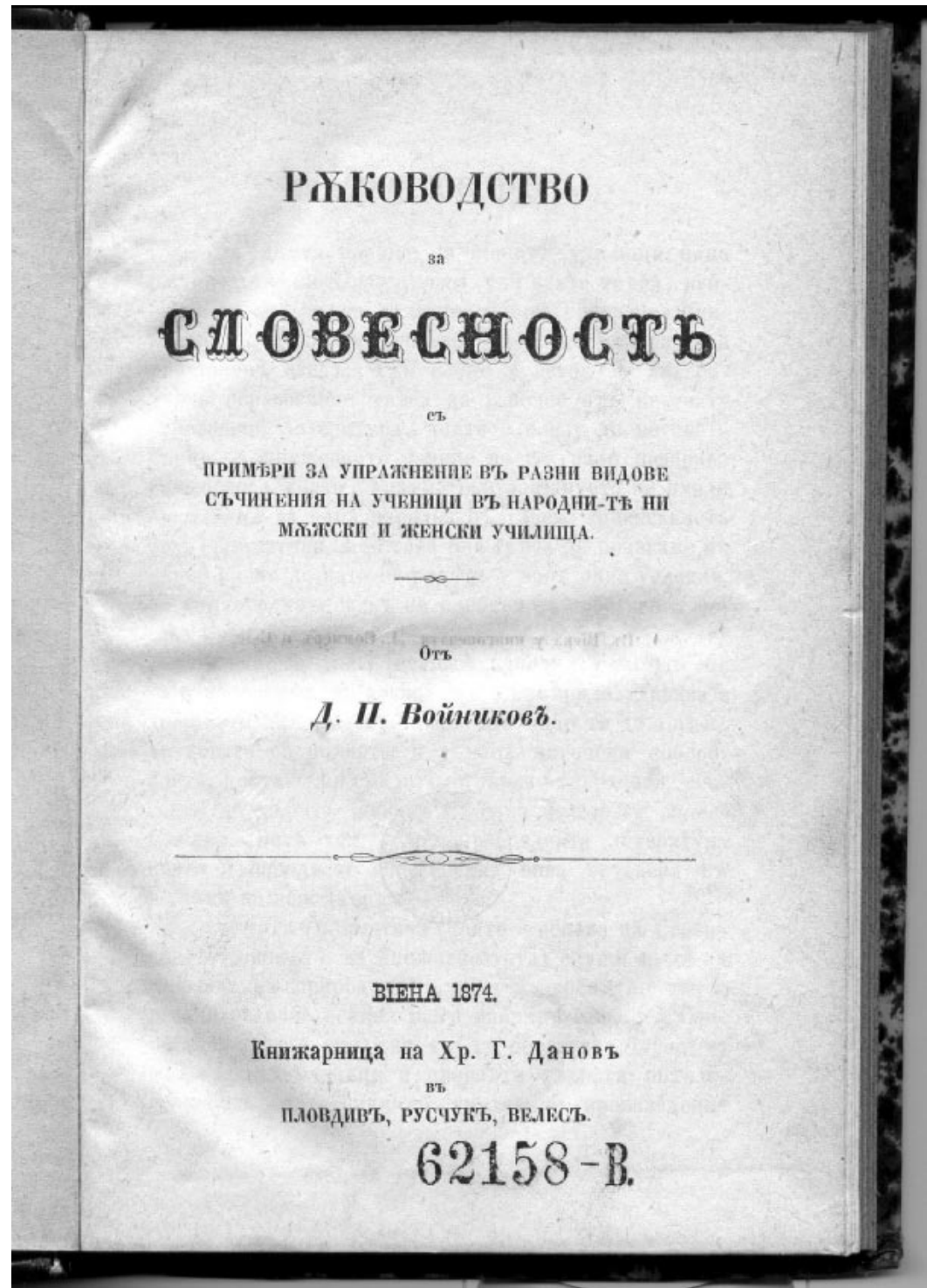

Abb. 5: Vojnikov, Dobri P.: Răkovodstvo za slovesnost s primeri za upražnenie v razni vidove săčinenija na učenici v narodnite ni măžski i ženski učilišta. - Viena [u.a.], Danov 1874. 332 S. Sign.: 62.158-B.Neu- 


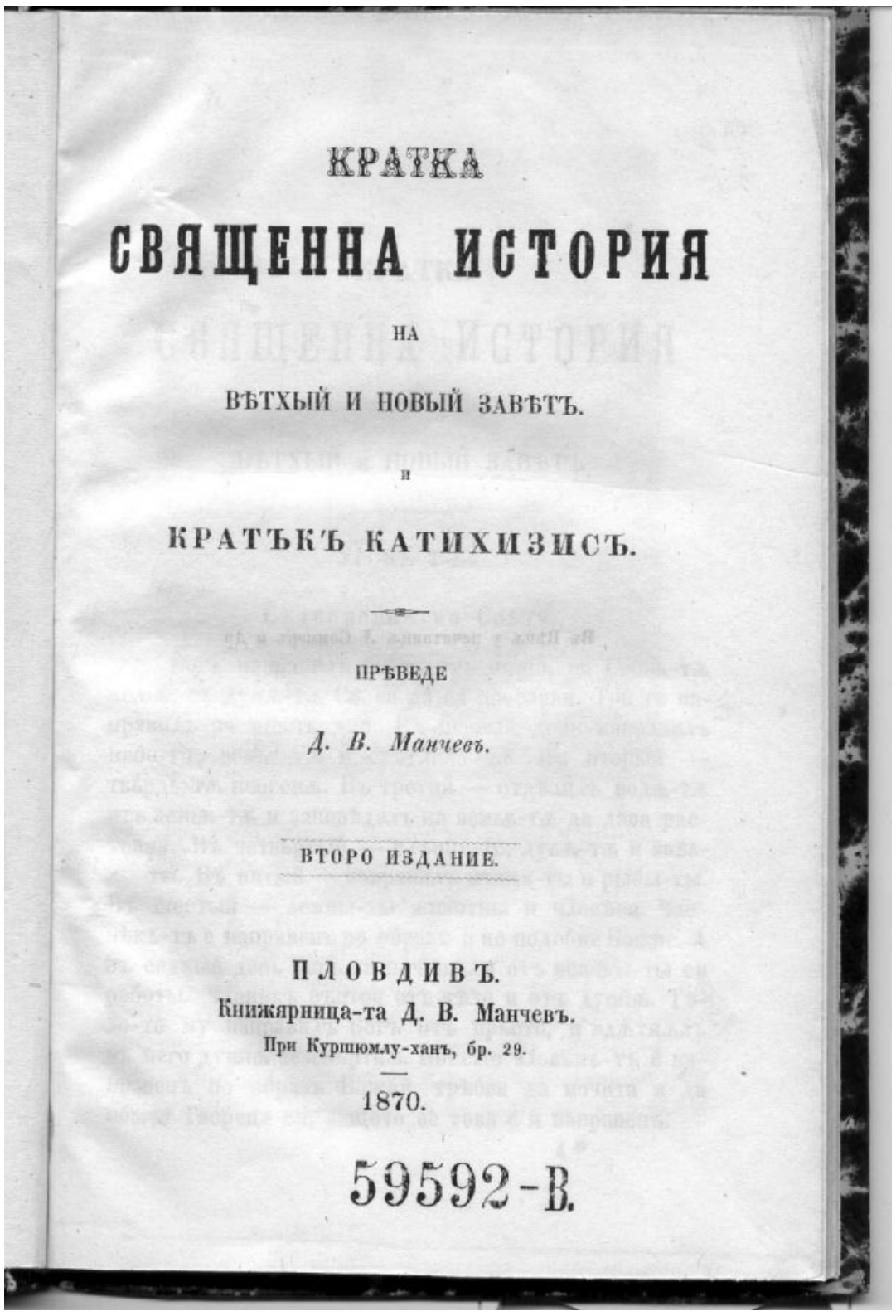

Abb. 6: Mančev, Dragan V.: Kratka svjaštenna istorija na větchyj i novyj zavět i kratăk katichizis. - Plovdiv, Mančev 1870. 80 S. Sign.: 59.592-B.Neu- 


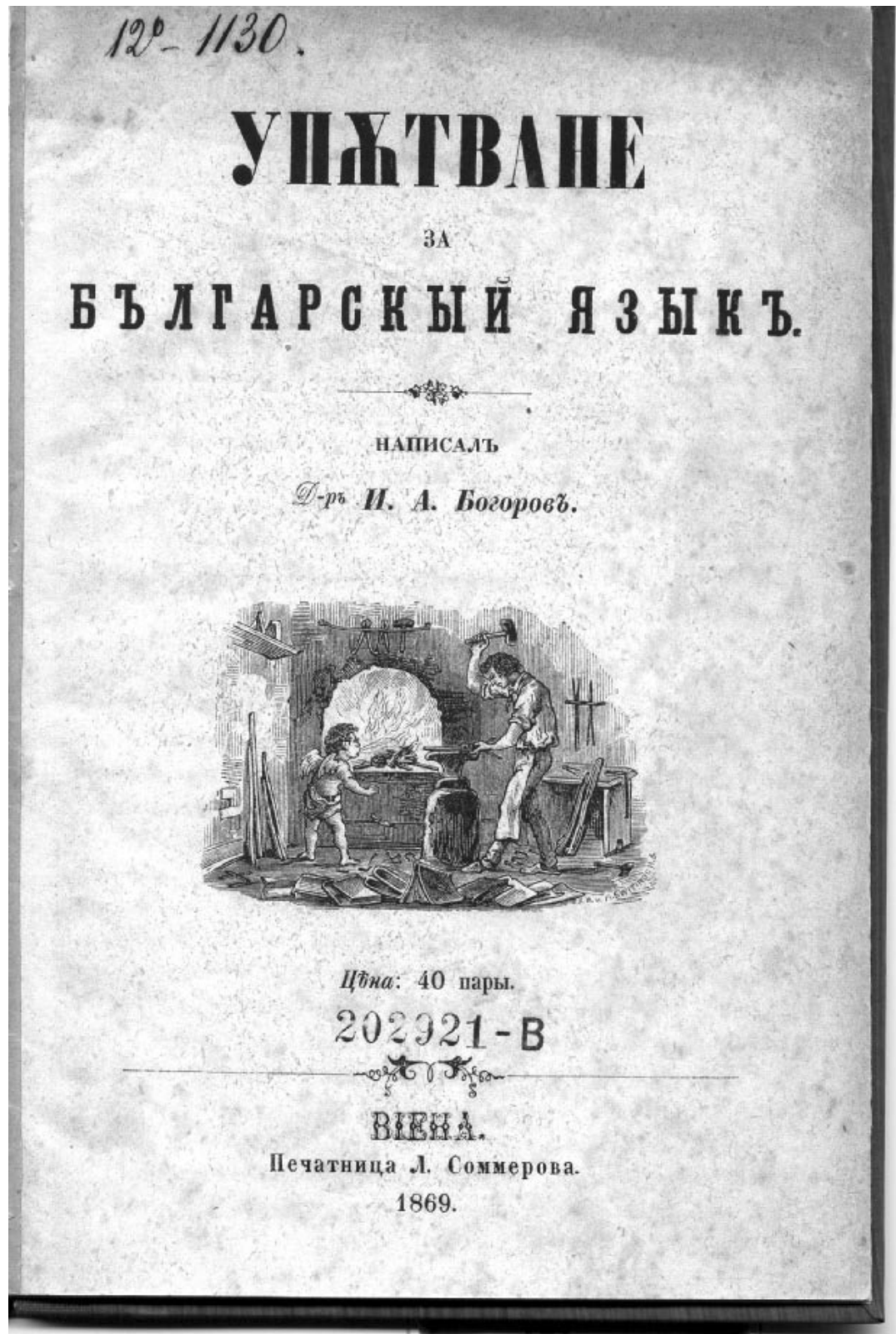

Abb. 7: Bogorov, Ivan A.: Upătvane za bălgarskyj jazyk. - Viena, Sommer 1869. 15 S. Sign.: 202.921-B.Neu- 


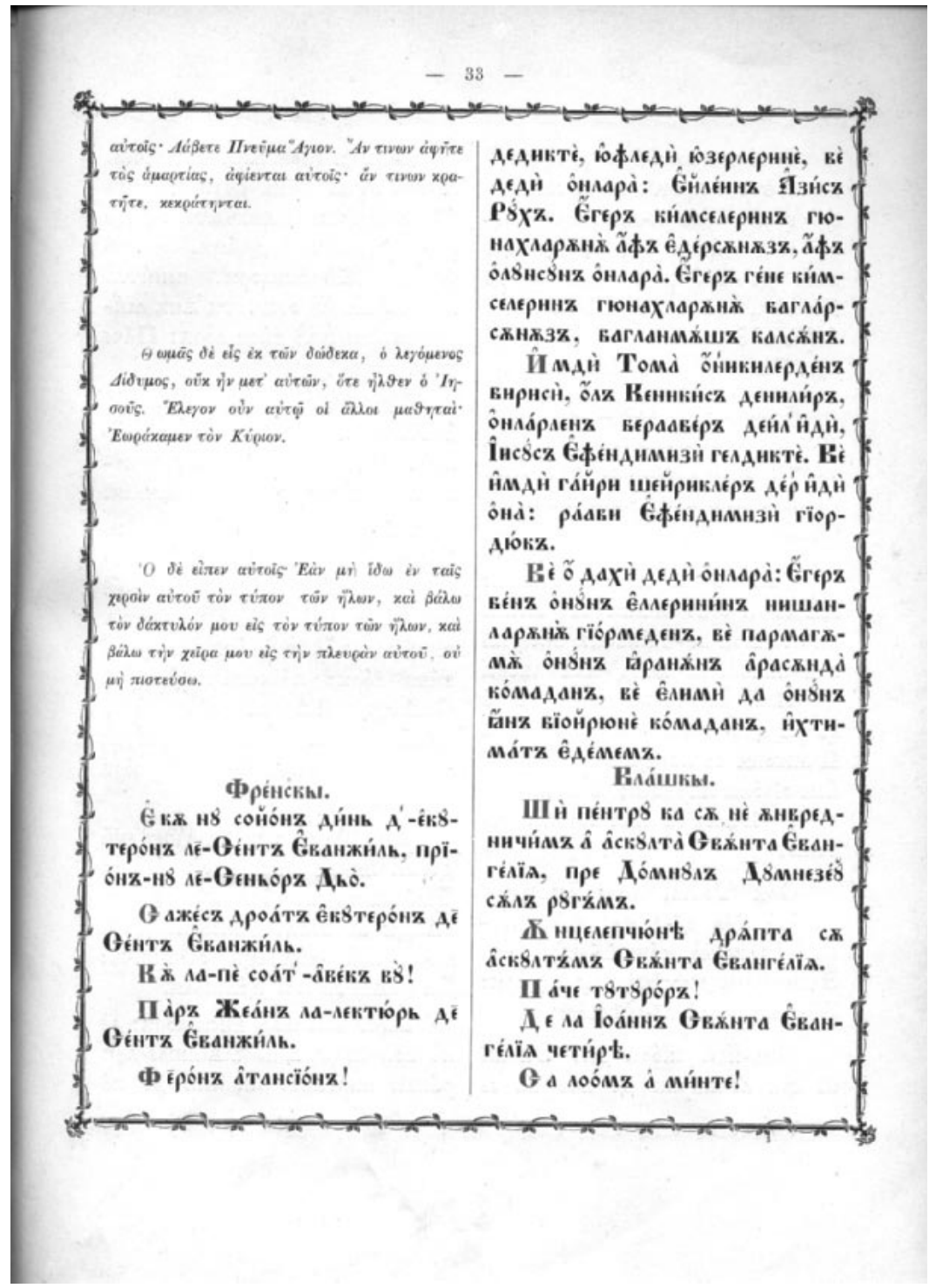

Abb. 8: Momčilov, Ivan N.: Cărkoven cvětnik. - Tărnovo, Momčilov 1869. 103 S. Sign.: 57.728-C.Neu-. Französische und rumänische Evangelienlesung in kyrillischer Schrift.

Karmen Petra Moissi

Abt. f. Sacherschließung u. Arbeitsgruppe Slavica

Österreichische Nationalbibliothek

Josefsplatz 1, 1015 Wien, Österreich

karmen.moissi@onb.ac.at 